\title{
La estructura de la comunidad deportiva: una propuesta comunicativa
}

\section{(The structure of the sporting community: a dialogical proposal)}

Francisco Javier LÓPEZ FRÍAS

Recibido: 4 de enero de 2014

Aceptado: 22 de octubre de 2014

\section{Resumen}

Este artículo se propone mostrar que la filosofía anglo-americana y la continental han de trabajar de forma conjunta dentro del área de la filosofía del deporte. Para ello, analizaré y aplicaré el concepto de la "comunidad comunicativa", que se encuentra en la ética del discurso de Apel y Habermas, en relación al deporte. Algunos autores han tratado de realizar esta tarea con anterioridad, por ejemplo, Raúl Sebastian Solanes, Robert L. Simon y William J. Morgan. Por lo tanto, analizaré sus propuestas de modo crítico. Al hacerlo, mostraré que hemos de luchar por conseguir una comunidad deportiva más integrativa que la propuesta hasta el día de hoy, con el fin de dar sentido al deporte contemporáneo.

Palabras clave: ética del deporte, filosofía del deporte, hermenéutica, comunidad comunicativa, comunidad deportiva.

\section{Abstract}

The main goal of this paper is to argue that Anglo-American philosophy and Continental philosophy should work together within the arena of the philosophy of sport. To do so, the concept "communicative community", which is found in Habermas' and Apel's discursive ethics, will be analyzed and applied to sports. As several authors, such as Raúl Sebastian Solanes, Robert L. Simon and William J. Morgan, have done this task before, I will critically analyze their proposals. In so doing, I will show that in order to make sense of contemporary sports we need a 
more integrative communicative sporting community than the ones proposed so far in the literature.

Keywords: sports ethics, philosophy of sports, hermeneutics, communicative community, sporting community.

\section{Filosofía continental y ética del deporte}

La filosofía del deporte actual es eminentemente filosofía -o ética- aplicada al deporte (López Frías 2012; 2014a). Tras unas fases iniciales puramente teóricas, la irrupción de Tras la Virtud de Alasdair MacIntyre y su propuesta de hermenéutica de las prácticas sociales influyó de tal manera en esta joven disciplina que acabó adoptando, primero, su clara vocación práctica y, segundo, su terminología básica-a saber: bienes internos y externos, así como la idea de que el deporte es una práctica social (López Frías 2014c). De este modo, la filosofía del deporte, cuyo paradigma principal pasó a ser el internalista, se convirtió en una disciplina en la que la reflexión va siempre encaminada a resolver problemas prácticos como el dopaje o la mercantilización del deporte. Tanto es así, que el filósofo Graham McFee (2004) llegó a afirmar que dentro de esta disciplina los únicos problemas que son verdaderamente filosóficos son los de tipo práctico. Si bien aquí no adoptaré una posición tan extrema como la de este autor, sí que reduciré mi discusión a esta tendencia mayoritaria-es decir, al ámbito de la ética deportiva-, dejando de lado otro tipo de discusiones de tipo ontológico o metafísico y que, a mi parecer, son tan valiosas como las éticas, pero están menos presentes en la actualidad dentro de esta disciplina.

Dado que la ética del deporte ha nacido en el ámbito de la filosofía anglo-americana, muy pocos autores han dialogado y recurrido a formulaciones filosóficas continentales para tratar de analizar los problemas con que se encuentra dicha disciplina. Es más, muy pocos filósofos de aquellos que introduciríamos en la categoría de "grandes figuras de la filosofía continental" ha publicado texto alguno referido al deporte. Entre las honorables excepciones se encuentran José Ortega y Gasset y Karl Otto Apel. En lo que se refiere a aquellos que han tratado de utilizar el pensamiento de autores clásicos de la filosofía continental para pensar los problemas relativos al deporte-utilizo esta expresión dado el carácter polémico que posee toda reflexión filosófica referida a un tema de una tradición tan anti-académica como el deporte-, cabe destacar a William J. Morgan, Jesús Ilundaín, Emanuele Isidori y Jan Vorstenbosch entre otros.

Morgan, por ejemplo, ha tratado de aplicar la ética discursiva de Jürgen Habermas con el fin de arrojar luz sobre los problemas éticos generados alrededor del deporte. Siguiendo la propuesta ética de Habermas, este autor norteamericano 
propone que la finalidad de una ética del deporte así concebida debe ser la de generar "comunidades prácticas más ilustradas" (Giulianotti 2005, pp. 60-61). Raúl F. Sebastián Solanes, realizando una interpretación del famoso artículo que Apel trata los temas de la ética y el deporte: "Die ethische Bedeutung des Sport in der Sicht einer universalistischen Diskursethik", se ha posicionado también a favor de una comunidad deportiva con un carácter más dialógico e ilustrado. Del mismo modo, Robert L. Simon (2004) también ha defendido una interpretación discursiva de la filosofía del deporte.

El artículo que aquí se presenta se dedica a analizar de modo crítico la idea de una comunidad deportiva más comunicativa e ilustrada-o democrática. El modo de proceder será el siguiente: primero, en referencia a la interpretación que Sebastián Solanes hace del texto de Apel, defenderé, junto con Simon, que el modo en que la ética del discurso concibe las prácticas sociales y su regulación normativa es la más adecuada a la hora de evaluar éticamente la práctica deportiva. No obstante, la lectura que Sebastián Solanes hace de Apel debe complementarse, pues defiende que la comunidad deportiva debe ser más comunicativa y cómo puede conseguirse este objetivo, sin embargo, no explicita quiénes son los miembros de dicha comunidad, algo que sí realizan tanto Simon como Morgan.

A consecuencia de ello, en segundo lugar, este artículo analizará las propuestas de estos dos autores. El objetivo final será mostrar que la interpretación equivocada que Morgan posee de la propuesta habermasiana le conduce a realizar una propuesta de comunidad deportiva demasiado reducida -"intramural". Todo lo contrario sucede en la obra de Simon, a quien su adecuada comprensión de la propuesta ético-discursiva le permite concebir la comunidad deportiva de un modo más amplio e inclusivo, similar al que se pretende defender en este artículo.

\section{La necesidad de una comunidad deportiva más comunicativa e ilustrada}

La idea de una comunidad deportiva más comunicativa al modo de los principios normativos expuestos por la ética discursiva de Karl O. Apel y Jürgen Habermas ha sido defendida por diversos autores como: Raúl Francisco Sebastián Solanes, William J. Morgan, y Robert L. Simon. Así, por ejemplo, el primero afirma de modo muy acertado que:

[e]s necesario que los deportistas [...] acepten las reglas de juego, consensuadas previamente por los afectados y una vez aceptadas como tales, éstos deberían asumir un «presupuesto trascendental recíproco», que implique no transgredirlas y no utilizar nada que sitúe a sus contrincantes en una situación de desigualdad o que pueda dejarles a ellos en una situación más ventajosa, pues todos los implicados deben competir en igualdad de oportunidades para que de este modo la obtención de la victoria sea más justa (Sebastián Solanes 2013, p. 88). 
En su interpretación del pensamiento de Apel, Sebastián Solanes se sitúa ante la posibilidad de concebir las relaciones que se establecen en la comunidad deportiva de dos modos distintos: (a) bien como competición por la victoria; o bien (b) como competición por la mejor prestación. Según el primer modelo, el deporte queda reducido al mero uso de estrategias cuyo principal fin es el de conseguir la victoria a toda costa. Esta es la mentalidad prevalente en nuestro mundo deportivo actual. No hay más que revisar las principales publicaciones científicas en torno al deporte para corroborarlo: la gran mayoría de ellas están dedicadas a estudios biomecánicos que pretenden comprender mejor el funcionamiento mecánico del cuerpo con el fin de mejorar el rendimiento de los atletas.

Desde que la filosofía del deporte surgiera como tal, ésta se ha dedicado a enfatizar la importancia del segundo paradigma, el de la victoria como mejor prestación. De este modo, uno de los padres de esta disciplina, Warren P. Fraleigh (1984), defiende que debemos concebir al oponente no como un obstáculo que hemos de superar a toda costa, sino más bien como un compañero de juego y un facilitador de nuestra actividad: alcanzar la excelencia física. El deporte es así un esfuerzo mutuo por lograr la perfección física.

Siguiendo esta tesis, la propuesta dialógica de Sebastián Solanes comprende la actividad deportiva como un diálogo que es llevado a cabo según los presupuestos contra-fácticos de la ética del discurso. En este tipo de diálogos, cada persona que presenta sus argumentos ante la comunidad debe ser vista como un compañero que busca la excelencia de un modo comunitario y no como un rival. El objetivo de ésta es llegar a una comunidad ilustrada o, más bien, excelente, en la que todos sus participantes puedan intervenir en el proceso argumentativo de defender con razones su posición. Lo cual, trasladado a términos deportivos, significa que todos los participantes puedan de tener un papel relevante en el logro de la excelencia comunitaria a raíz de la búsqueda de sus metas. Cuando dos deportistas dan lo mejor de sí, ambos se benefician, pues encuentran en el otro el modo de alcanzar su máximo rendimiento. Hay múltiples ejemplos como estos en la historia del deporte, por ejemplo, los duelos entre Magic Johnson y Larry Bird, o entre Lionel Messi y Cristiano Ronaldo.

Esta propuesta se muestra como una concepción normativa novedosa y muy fructífera dentro de la filosofía práctica del deporte. No obstante, es necesario aclarar, al menos, dos cuestiones para que sea totalmente practicable. Primero, tendríamos que ver cómo se producen los diálogos dentro de la comunidad deportiva. Si bien es cierto que la práctica deportiva se estructura y articula gracias a diversas instituciones dentro de las cuales pueden desarrollarse debates prácticos sobre las cuestiones normativas que les preocupan: la trampa, la corrupción, el dopaje... Sin embargo, como ha defendido Graham McFee (2009), en un ámbito tan eminentemente práctico como el deporte, los diálogos también se pueden producir de otro modo, a saber, a través de la misma práctica. 
Así, por ejemplo, como muestra Jonathan Wilson (2008) en Inverting the Pyramid, la historia del fútbol puede entenderse como un diálogo entre las diversas tradiciones tácticas: el dibujo táctico se ha ido invirtiendo desde estrategias que poseían una amplia línea ofensiva hacia el desarrollo de otras con una base defensiva más sólida. Este cambio se ha producido por la excesiva importancia que poco a poco se le ha dado a la victoria sobre lo vistoso del juego o la calidad del mismo. Así, se ha desarrollado un debate, aún existente entre aquellos que proponen un fútbol más vistoso y aquellos que optan por ser más efectivos. Este diálogo no se ha llevado a cabo por medio de palabras, sino a través de las decisiones tácticas de los entrenadores en los enfrentamientos mutuos.

Una segunda cuestión que necesita aclarar esta propuesta dialógica en ética del deporte es la de acotar claramente quienes componen la comunidad deportiva. Este último término no queda nada claro, pues si bien parece referirse a aquellos directamente envueltos en la práctica deportiva, habría que aclarar qué es eso de "estar envuelto directamente" en una práctica; ¿nos referimos con ello sólo a aquellos que la practican, es decir, a los deportistas? ¿O también a los que influyen en la actuación de aquéllos, como los entrenadores o los directivos? ¿No influye también el público en la actuación de los deportistas? ¿Y qué decir de la prensa, los académicos, y la opinión pública que discuten sobre la práctica deportiva y generan su imagen pública? ¿No tienen también los Estados cierta importancia en el deporte a la hora de iniciar y facilitar a los más pequeños su entrada en el mundo deportivo a través de construir instalaciones, ofrecer incentivos...?

Siguiendo el principio máximo de la ética discursiva, Robert L. Simon (2004) afirma que todos los afectados por las consecuencias de aquello que acontece en el deporte deberían ser tenidos en cuenta. Así pues, este concepto queda extendido hasta casi el total de la sociedad. La propuesta dialógica de Sebastián Solanes no deja clara ninguna de estas cuestiones. Sin embargo, tanto Morgan como Simon sí han tratado de profundizar en ellas. Comenzaremos por analizar la propuesta de Morgan, quien da respuesta a la primera de nuestras preguntas en su artículo de 2012 "Broad Internalism, Deep Conventions, Moral Entrepeneurs, and Sport", y a la segunda en su libro Leftist Theories of Sport publicado en 1994.

\section{La propuesta hermenéutica de Morgan: convencionalismo internalista}

\subsection{Las convenciones constitutivas no tienen por qué ser verbalizadas}

Denominaré a la propuesta de Morgan como "convencionalismo internalista" (López Frías 2014b). Ésta se encuentra principalmente en su libro Why sports morally matter y ha sido extendida en su artículo "Broad internalism, deep conventions, moral entrepreneurs, and sport", cuyo fin principal es el de revisar las bases 
teóricas sobre las que se asienta el internalismo en ética del deporte con el fin de que "demos sentido al escenario del deporte contemporáneo" (Morgan 2012, p. 89). En este artículo afirma que aunque ésta es una tarea necesaria, sin embargo, la propuesta de Morgan es equivocada.

Según la interpretación que Morgan, el internalismo pretende formular una interpretación del deporte basándose en sus elementos propios distintivos o intrínsecos. Éstos, al pertenecer de forma exclusiva al deporte, son "criterios que poseen un fundamento racional independiente de cuestiones culturales, lingüísticas o pragmáticas" (Simon 2004, p. 125). Para ello, los autores internalistas tratan de mostrar las diferentes capacidades humanas que todos los seres humanos deberían ejercitar con el fin de realizar los bienes y excelencias propias del deporte. Según la mayoría de las propuestas internalistas, estos criterios normativos están relacionados con la lucha por la excelencia física dando lugar a lo que se denomina como "el espíritu del juego" o "la integridad del deporte". El fracaso en cumplir con estos criterios supone una traición a los principios del deporte.

Así, según Morgan, el internalismo es concebido como una propuesta deontológica preocupada por el cumplimiento de aquellos principios que determinan el propósito del deporte. En tanto que, a juicio de Morgan, la propuesta internalista es exclusivamente deontológica, ésta debe complementarse con otros elementos con el fin de que no se deje de lado la situación histórica y social aportada por los contextos sociales a los que pertenece el deporte. Si el deporte queda desarraigado de ellos, la discusión racional sobre los temas que preocupan a la comunidad deportiva no es posible. Por ello, Morgan trata de complementar el internalismo con un conjunto de convenciones que él denomina, siguiendo los términos de Andrei Marmor, como "convenciones constitutivas".

Morgan, al proponer su teoría convencionalista, no afirma que el internalismo deba ser rechazado, sino que tiene que ser complementado con el fin de que sus análisis y propuestas normativas estén situadas histórica y socialmente. Esta es la razón por la que he denominado el pensamiento de este autor como "convencionalismo internalista", pues en contraste con aquellos autores, por ejemplo, los teóricos marxistas del deporte que defienden que el deporte se rige por las convenciones sociales dominantes en el global de la sociedad-por ejemplo, los principios y valores establecidos por las clases dominantes para mantener su poder-, propone que las convenciones que deben explicar y regular la práctica deportiva son relativas a la naturaleza y bienes intrínsecos propios del deporte en sí mismo (Morgan 2012, p. 71). De este modo, los análisis filosóficos del mismo quedan contextualizados y enraizados en el momento en que éstos se desarrollan.

Según Morgan, las convenciones constitutivas proporcionan el contexto en el que el acuerdo y la argumentación racional son posibles. El hecho de que éstas sean condiciones de posibilidad de la argumentación muestra que no son fruto de la 
mera agregación de opiniones, es decir, del acuerdo puntual respecto a un tema, sino que poseen un carácter racional que se fundamenta en el hecho de que todos y cada uno de los miembros de la comunidad las comparten, sea de un modo consciente o no-lo cual recuerda a ese fundamento o "roca dura" wittgensteniano en los que se sustentan nuestras creencias (Wittgenstein 2000). Así pues, estas convenciones pueden no ser verbalizadas o dichas de modo explícito pero permanecen en el trasfondo de las acciones, decisiones, y argumentos que encontramos dentro de la comunidad deportiva.

De un modo muy hermenéutico, para el convencionalismo de Morgan, la tarea de la filosofía del deporte es la de descubrir esos elementos constitutivos que están"tras las espaldas" de los protagonistas de la comunidad deportiva. De este modo, podemos ofrecer una respuesta a la primera cuestión que quedaba sin responder en la propuesta comunicativa de Sebastián Solanes. Según Morgan, estas convenciones constitutivas son la clave para comprender el fenómeno del deporte, pero no tienen por qué estar formuladas lingüística y explícitamente. Por ejemplo, nuestra fascinación por los equipos más desfavorecidos que hacen un buen papel-que en el ámbito anglo-americano se denominan como "underdog"-muestra la influencia que aún tiene en nosotros el ideal del atleta amateur del siglo XIX y nuestro recelo a dejar que el comercialismo entre de modo pleno en el deporte.

\subsection{Los miembros de la comunidad deportiva: protagonistas del debate}

Morgan fundamenta su propuesta convencionalista en una concepción dialógica de las comunidades prácticas, ya que defiende que las convenciones constitutivas proporcionan la base que posibilita tanto el debate, como el acuerdo racional. De este modo, el análisis racional de la actividad deportiva sólo es posible si se fundamenta en ese sustento que proporcionan las convenciones constitutivas. En relación a este modo de concebir cómo funcionan la argumentación y el debate, Morgan establece una afirmación muy fuerte teóricamente hablando:

[1]a resolución racional de los conflictos normativos relativos al propósito del deporte es, por lo tanto, posible en mi propuesta sólo si estos conflictos son de un tipo intramural en vez de extramural. (Morgan 2012, p. 76)

Según Morgan, la filosofía del deporte tiene que proceder de un modo "intramural". Si el debate sólo es posible sobre el trasfondo de convenciones constitutivas compartidas de modo interno dentro de cada práctica; y si éstas no pueden ser transculturales al estar siempre referidas a un contexto histórico particular; entonces se rechaza la posibilidad de buscar principios transculturales-es decir, de dialogar más allá de las fronteras marcadas por cada contexto social particular-que se encuentran en todas las prácticas sociales. Debido a esta concepción intramural, 
Morgan afirmará que el debate relativo a las comunidades deportivas debe ser desarrollado y protagonizado por "aquellos directamente envueltos en la práctica deportiva" (Morgan 1994, p. 234).

En el último apartado de su Leftist Theories of Sport Morgan distingue entre agentes primarios y secundarios (1994). En la primera categoría incluye a aquellos que practican deporte como tal, es decir, los atletas. En la segunda categoría incluye a los entrenadores, espectadores, críticos, periodistas, y estudiosos del deporte. Sin embargo, establece un requisito básico para que estos últimos puedan participar de lleno en los debates: "fidelidad a los bienes internos a la práctica, la cual, por supuesto, presupone más que un conocimiento de pasada y la apreciación de la complejidad de la práctica" (Ibíd, p. 236).

Así, ser miembro de la comunidad deportiva significa, a juicio de Morgan, pertenecer a una "fraternidad particular", pues todos sus miembros comparten unos bienes comunes y una identidad. Tanto es así, que Morgan afirma que a los miembros de "las prácticas deportivas debe permitírseles hablar su propio lenguaje, [...] cuyas nociones clave $[\ldots]$ se refieren a bienes y virtudes que no tienen similitud con el lenguaje de [otras] instituciones" (Ibíd, p 242). Sólo aquellos que, a través del contacto con los agentes primarios, sean capaces de adquirir ese lenguaje propio de la práctica deportiva pueden participar en los debates de la comunidad deportiva. Los participantes y conocedores de la práctica deben los protagonistas de los debates (Ibíd, p. 240). No obstante, ¿quiénes pueden considerarse como tal? Esta es una pregunta que podemos responder perfectamente desde la ética discursiva. Si Morgan la responde de un modo reductivo es porque, primero, rechaza esta propuesta ética y, segundo, la comprende de un modo equivocado.

\section{Las bases hermenéuticas del convencionalismo internalista: el rechazo de Habermas}

Como se ha mostrado en la sección anterior, el punto de partida de la filosofía de Morgan es el "nosotros" compuesto por la comunidad particular que es el deporte. Las teorías hermenéuticas de Rorty, Habermas, y Gadamer están a la base de esta propuesta. Todos estos filósofos comparten el presupuesto hermenéutico fundamental que defiende que siempre nos encontramos de modo ineludible en una situación dialógica no sólo con los otros, sino también con la tradición y el ámbito social del que formamos parte. Tal y como muestra Morgan, siempre estamos influenciados por un conjunto de convenciones y principios heredados de los contextos de los que formamos parte.

Aunque Gadamer, Rorty y Habermas comparten este principio dialógico, existe una diferencia fundamental entre ellos: el propósito principal de la filosofía de Habermas es adquirir criterios objetivos a través de los cuales poder distinguir 
entre aquellos acuerdos que son alcanzados de modo racional y aquellos que no. Habermas, en tanto que filósofo hermenéutico, acepta la existencia de diversos "mundos de la vida" o contextos sociales que tienen sus códigos particulares-o lenguajes específicos. No obstante, su propuesta dialógica concibe el lenguaje ordinario como un medio general de comunicación que tiene la capacidad de llevarnos más allá de códigos particulares (Habermas 1998, p. 119). Por ello, los diversos lenguajes son traducibles entre sí, a pesar de las dificultades y complejidades que ello plantea. Esto muestra que, más allá de las diferencias, existe un hilo o elementos comunes a los que podemos apelar para entendernos y a través de los que debatir. La comprensión trans-contextual es posible.

Esta diferencia entre la propuesta normativa de Habermas y las de Rorty y Gadamer es crucial desde un punto de vista normativo. Por ello es por lo que se denomina a la propuesta del primero como "hermenéutica crítica de las prácticas sociales". Ésta es, utilizando las palabras del filósofo Richard J. Bernstein (1982), "la diferencia determinante", pues pone en cuestión la propuesta de Morgan de que la filosofía del deporte debe proceder de un modo intramural pues no existen principios comunes compartidos entre las diversas prácticas sociales. Dada esta diferencia de posicionamiento, Morgan dedica un capítulo completo de su libro Why Sports Morally Matter y un artículo titulado "Social Criticism as Moral Criticism: A Habermasian Take on Sports" a la tarea de mostrar que la ética de Habermas no sería válida para realizar un análisis normativo del fenómeno deportivo. En éstos, Morgan concibe la teoría ética de Habermas del siguiente modo:

[1]a teoría ética de Habermas nos proporciona [...] pistas importantes para desentrañar la difícil situación moral del deporte actual. Sin embargo, [ésta] se torna problemática y acaba generando complicaciones que había aclarado previamente cuando trata de separar las cuestiones éticas relativas al bien de las cuestiones morales referidas a lo correcto con el fin de privilegiar estas últimas sobre las primeras (Morgan 2002, p. 237)

Es cierto que Morgan acepta ciertos puntos de la filosofía de Habermas. No obstante, su recepción del pensamiento de Habermas deja bastante que desear. Por un lado, rechaza ese elemento que marca la diferencia con respecto al resto de teorías éticas hermenéuticas, incluso cuando éste proporciona un elemento crítico determinante de gran potencial a la hora de analizar el fenómeno deportivo. Por otro lado, este rechazo surge porque Morgan ha malinterpretado el pensamiento de Habermas por su necesidad de mantenerse del lado del internalismo ético en filosofía del deporte. Las siguientes dos secciones se dedicarán a analizar estos puntos para mostrar que la concepción que Morgan elabora de la comunidad comunicativa debería ser de otro modo con el fin de dar sentido al mundo del deporte actual. 


\subsection{Principios morales y cuestiones éticas}

Morgan defiende que Habermas se equivoca al defender que el fin principal de la filosofía moral debe ser el de encontrar principios morales que de modo transcontextual pueden aplicarse a todos los contextos sociales-con sus códigos éticos particulares-posibles (Morgan 2002, p. 289). Según este filósofo del deporte norteamericano, para que la argumentación sea posible, ésta debe realizarse a través de los códigos compartidos por los miembros de cada comunidad. Así, los principios transcontextuales no tienen sentido. Un debate basado en ellos sería demasiado abstracto y no proporcionaría las claves para comprender una práctica tan concreta y particular como el deporte (Ibíd, p. 292).

Para Habermas, la ética contemporánea necesita de principios universales transcontextuales con los que enfrentarse a los problemas morales generados por la actual sociedad globalizada. No vivimos en un mundo compuesto por pequeñas comunidades cerradas en sí mismas, sino en uno globalizado en el que todo está interconectado. En este contexto global los proyectos filosóficos que se sustentan en visiones internalistas de los diversos contextos y comunidades están abocados al fracaso. A pesar de que éstos existen, y hay que reconocerles su peso, los seres humanos seguimos compartiendo una serie de características básicas que nos hacen iguales. Aplicando este presupuesto universalista y transcontextualista al caso del deporte, la propuesta internalista resulta incompleta a la hora de explicar y comprender la naturaleza de un deporte contemporáneo que es un fenómeno global a todas luces -quizás, el fenómeno social más global de todos.

Esto no significa que las propuestas internalistas sean completamente equivocadas y que carezcan de valor crítico. Lo que sucede es que, según la interpretación de Morgan, éstas no son suficientemente críticas ya que defienden que los análisis normativos de la práctica deportiva deben cimentarse en los principios internos y exclusivos de la práctica deportiva pues, utilizando la terminología de Morgan, la argumentación y los acuerdos racionales dentro de esta comunidad deportiva son sólo posibles a partir de principios compartidos de modo interno en dicha comunidad. Por el contrario, la propuesta filosófica de Habermas se fundamenta en ciertos principios que pueden ser encontrados en todos y cada uno de los contextos existentes - pero una cosa es que se fundamente, y otra muy distinta es que se reduzca a ellos.

Atendiendo a lo afirmado hasta ahora sobre la hermenéutica crítica de Habermas, surge la siguiente pregunta: ¿cómo podemos defender la existencia de criterios objetivos a la vez que aceptamos que nuestro mundo está caracterizado por una pluralidad de comunidades, prácticas sociales, y concepciones del bien que tienen su propia especificidad? Con el fin de responder a estar cuestión, Habermas recurre a los presupuestos del habla. Éstos sirven como criterios para distinguir 
entre el consenso racional genuino y el que es fruto de una comunicación distorsionada. Toda persona que quiera ponerse a argumentar debe cumplir con éstos a riesgo de dejar de ser racional si no lo hace. Estos principios son hermenéuticos en tanto que son inevitables para cualquier individuo con capacidad lingüística-están siempre ahí, tras sus espaldas, en el trasfondo-, y a su vez son críticos porque generan una serie de obligaciones y relaciones de orden moral que deben serle reconocidas a todos los seres humanos en tanto que seres capaces de argumentar (Cortina 2008).

Estas relaciones morales que emergen de la naturaleza lingüística de los individuos se sitúan en lo que Habermas denomina como el ámbito moral de la razón práctica. Como explica en Facticidad y validez, el ámbito práctico de la razón se divide en tres esferas: pragmática, que está relacionada con la búsqueda de las estrategias adecuadas para alcanzar metas y objetivos individuales; ética, relacionada con el desarrollo comunitario de planes de vida; y moral, que tiene que ver con los principios que regulan la convivencia humana desde el punto de vista de la justicia. Según la interpretación que Morgan hace del pensamiento de Habermas, los análisis normativos del deporte deben situarse en la esfera ética, pues la comunidad deportiva es generada, al fin y al cabo, a raíz del establecimiento de una cierta concepción del bien que tiene sus propias virtudes, bienes, y principios.

Para Morgan, estas diversas esferas serían impermeables, de modo y manera que basta con referir la ética del deporte al ámbito ético para realizar un análisis adecuado del deporte. Esto es lo que defiende el internalismo ético en filosofía del deporte. Así, por ejemplo, en el debate en torno al dopaje, dos autores claramente internalistas como son Angela Schneider y Robert Butcher defienden que los argumentos relativos al daño físico, la coacción, y la influencia del dopaje sobre la sociedad son externalistas, pues se fundamentan en principios que no son propios de la práctica deportiva-“daño físico" y "coacción" (Schneider y Butcher 2000, pp. 187 y 195).

Sin embargo, en contra de esta interpretación realizada por Morgan. La teoría del discurso de Habermas no concibe esta división tripartita de la razón práctica como si cada nivel fuera un compartimento estanco, sino de un modo poroso. Es decir, siempre existe relación entre las diversas esferas. El hecho de que el deporte sea, como es imposible negar, un contexto social con ciertos principios, valores, y virtudes propios no excluye el hecho de que los razonamientos pragmáticos y morales tengan también un papel esencial en los análisis del deporte. Así, por ejemplo, como reconoce Sebastián Solanes (2012) siguiendo a Adela Cortina (2010), ámbitos como el deporte, la política, y la bioética no puede renegar del uso estratégico de la razón, pues ésta es también esencial para su cometido. En el caso particular del deporte, ¿podríamos concebirlo sin dejar lugar a la estrategia y al cálculo de medios para alcanzar ciertos fines? ¿No está este espíritu pragmático a la base de la vida del deporte moderno? 
Para seguir profundizando en esta idea de la naturaleza porosa de los ámbitos de la razón práctica, analicemos lo siguiente: los deportistas tienen como principal meta alcanzar la victoria. Para ello, tienen que entrenarse para mejorar su técnica y elegir estrategias con el fin de incrementar su capacidad de salir victoriosos. Éstas pertenecen al uso pragmático de la razón pero también poseen implicaciones éticas y morales pues, por ejemplo, algunas técnicas como el dopaje pueden atentar contra valores-éticos-propios de la práctica deportiva y contra principios morales como la justicia o la autonomía. De este modo, debe quedar claro que aunque existe una distinción de usos de la razón práctica, ésta, al fin y al cabo, es una sola entidad. Así pues, los tres usos de la razón son partes de un continuo: la razón práctica humana. Todo análisis normativo es realizado por ella. Por lo tanto, no tiene sentido alguno reducir los análisis normativos de una práctica concreta como el deporte a una sola esfera.

Morgan acusa de externalista a cualquiera que use la filosofía habermasiana para explicar el fenómeno deportivo. Según el externalismo, el deporte debe ser explicado según los principios que rigen el global de la sociedad. Para Morgan, apelar a principios morales que todos compartimos supondría obviar la especificidad de la práctica deportiva al analizarla a la luz de principios generales que pueden ser aplicados a cada práctica. No obstante, la correcta lectura de la filosofía habermasiana, como la proporcionada por Simon (2004), muestra que: primero, aquel que utiliza la ética habermasiana para pensar el fenómeno del deporte no cae en el externalismo, pues los análisis normativos no se basan exclusivamente en uno de los usos de la razón. Segundo, que la dualidad internalismo-externalismo debe abandonarse en ética del deporte en favor de una posición intermedia, más compleja, que evite este tipo de concepciones dualistas de la realidad (López Frías 2014b). Siguiendo a R. Scott Kretchmar, el dualismo en filosofía conduce a callejones sin salida que sólo pueden ser solucionados a través de la complementación y la integración (Kretchmar 2013, p. 275).

Estar dentro de este esquema dualista, y no querer abandonar el lado del internalismo, aboca a Morgan a malinterpretar el pensamiento de Habermas, lo cual tiene consecuencias prácticas importantes relativas a la interpretación de los problemas-globales-que acechan a nuestro deporte. En el siguiente apartado analizaré cómo esta confusión determina que Morgan defienda que sólo los agentes primarios y los agentes secundarios que posean un conocimiento adecuado de la práctica deben entrar en los debates normativos respecto al deporte.

\subsection{La composición de la comunidad deliberativa deportiva}

Contra la interpretación que Morgan realiza de Habermas, la ética discursiva no defiende que debamos tratar de alcanzar una verdad moral que sea alcanzada de modo universal y objetivo, sino que Habermas reclama que hemos de tratar de 
modular los principios de la acción y la racionalidad comunicativa dentro del horizonte de nuestras prácticas sociales con el fin de construir situaciones dialógicas lo más racionales posibles. Para Habermas, estos principios comunicativos no pertenecen a un mundo trascendental donde se encuentra la llamada "verdad práctica", sino que, como afirmé con anterioridad, son ciertos presupuestos fácticos que todos los individuos han de reconocer porque están ahí de modo inevitable.

Estos presupuestos fácticos están relacionados con las capacidades comunicativas de los seres humanos, las cuales sirven como presupuestos de la argumentación. Los individuos que participan en el debate público han de aceptar ciertas reglas que se derivan de dichas capacidades comunicativas y presupuestos de la argumentación (Habermas 1998, 65). Como consecuencia, la ética del discurso se ocupa principalmente del modo en que se diseñan el debate público y en el papel que juegan las instituciones en tal proceso, ya que son éstas las que deben proporcionar las condiciones adecuadas para que el debate se desarrolle de un modo más racional (Habermas 1996, 371-372; 380).

A través de este debate se consigue la unión de las tres esferas de la razón práctica: la moral, la ética, y la pragmática. En los debates configurados según los principios morales, encontrados de modo hermenéutico apelando a nuestra naturaleza comunicativa, los individuos entran a debatir llevando sus propias concepciones éticas del bien, y tratando de alcanzar acuerdos desde los que regular la convivencia del mejor modo posible. Para ello siempre es necesario un cálculo pragmático que evalúe los recursos disponibles y el modo más correcto de organizarlos (Cortina 2010). Así, el tan criticado ideal de la comunidad ideal de comunicación de Habermas no es una utopía, sino un ideal regulativo que muestra cómo deben organizarse los debates reales que tienen lugar dentro de nuestras comunidades.

Como defiende Simon (2004), en los debates organizados según la propuesta discursiva, los individuos no son obligados a abandonar sus concepciones del bien-al modo de los artificios ralwsianos del velo de la ignorancia y la posición original. El uso ético de la razón, las convenciones éticas propias de cada uno, en contra de lo que defiende Morgan, juega un papel esencial en el pensamiento habermasiano. Los principios morales no nos abstraen de él, sino que establecen cómo deben regularse los debates en los que, precisamente, ponemos en juego estas convicciones. En ese sentido, Habermas formula el famoso principio de la ética discursiva:

[t]oda norma válida debe satisfacer la siguiente condición: que puedan ser aceptadas por todos los afectados (y preferidas a las consecuencias de las posibles alternativas conocidas) las consecuencias y las consecuencias secundarias que, para satisfacer los intereses de cada individuo se seguirían (previsiblemente), en el caso de que fuera seguida universalmente (Habermas 1983, p. 75-76). 
Sólo si el debate se realizar de acuerdo con este principio podremos decir que el acuerdo alcanzado no estará basado en razones egoístas o en presiones de poder, sino en el verdadero consentimiento racional de los afectados. De este modo, el grupo de los individuos que deben participar en los debates normativos en torno al deporte debe incrementarse y llevarse más allá de lo que Morgan considera como agentes de primer y segundo nivel. Todos aquellos afectados por las consecuencias de las decisiones de estos debates han de participar, al menos virtualmente-teniendo en cuenta su opinión a la hora de tomar las decisiones y diseñar las normas. En un fenómeno tan globalizado como el deporte, el total de los afectados por las consecuencias de éste se extiende hasta casi el global de la población. Por ejemplo, el informe Miller sobre los hábitos de los ciudadanos norteamericanos afirma que el 98\% de los americanos tienen algún tipo de relación con el deporte (cit. por Simon 1991). De igual modo, la realización de eventos deportivos es financiado por todos, de modo que el global de la población debe ser consultada. A diferencia de lo que se ha hecho en Río con los Juegos Olímpicos de 2016, imponerlos a la población, el ayuntamiento de Oslo recientemente preguntó a la población si querían albergar los Juegos Olímpicos de Invierno de 2022 a través de una votación-que acabó resultando en un "no" mayoritario.

En debates de este estilo, en los que todos los participantes entran a formar parte de ellos con sus propias convicciones bajo el requisito de aceptar ciertas normas del discurso, tal y como trata de mostrar Simon (2004), la discusión entre los internalistas convencionalistas-intramurales-y los internalistas "deontológicos"-tal y como los llama Morgan-se disuelve casi completamente. De lo que se trata es de encontrar ciertos criterios objetivos que nos permitan tanto identificar a los participantes en los debates relativos a la comunidad deportiva, así como los principios que debe regir a éstos. Esta tarea es de búsqueda, deontológica, de principios objetivos y transcontextuales. Sin embargo, cada cual entrará en los debates portando sus propias convicciones, valores y visiones del deporte propias. Éstas no se abolen, sino que se ponen en discusión de un modo organizado con el fin de organizar de un modo mejor y más ético la comunidad deportiva. Todas las esferas de la razón prácticas son parte de un mismo proceso.

Recurramos, por ejemplo, al caso del debate sobre el dopaje. La propuesta de Habermas nos invita a luchar por una situación dialógica en la que todos los afectados por las consecuencias de las reglas anti-dopaje puedan participar en igualdad de condiciones; éste debe ser un debate de iguales entre iguales. Así, por ejemplo, la ley del silencio descrita por Verner Møller (2009) en sus obras, que prohíbe a los deportistas hablar abiertamente sobre el dopaje a riesgo de ser castigados, debe ser combatida a través de establecer espacios públicos en los que el tema se trate de un modo público y abierto. Además, tal y como ha mostrado el investigador Angelo Corlett (2013) y su equipo también debería tenerse en cuenta la posición de aque- 
llos que subvencionan con su trabajo y con sus cotizaciones los tratamientos médicos a los que los deportistas dopados tendrán que someterse una vez acabadas sus carreras. Lo cual extiende la "comunidad comunicativa deportiva" hasta los mismos Estados y a todos sus componentes, al menos en los estados sociales de bienestar en los que la sanidad es pública.

Una vez "sentados en la mesa" los diversos interesados en tratar y solucionar el problema del dopaje, cada cual debatirá a partir de su modo de concebir el deporte y de los intereses y valores que le constituyen. Así, por ejemplo, la Asociación Mundial Antidopaje portará su bandera de la tolerancia cero contra el dopaje-elaborada a partir de los mimbres proporcionados por la ideología amateur decimonónica. Por su parte, los representantes de las ligas profesionales ofrecerán una visión del deporte menos pura, más ligada al espectáculo y el entretenimiento que, al fin y al cabo, son los que les hacen ganar dinero. Por último, deportistas y seguidores se posicionarán de muy diversos modos dependiendo de la concepción del deporte que alberguen. Para unos, el deporte será una cuestión de probar quién es el mejor dotado genéticamente o para un premio a la constancia y el entrenamiento, mientras que para otros no será más que una expresión de nuestro esfuerzo por ser cada vez mejores-más altos, más rápidos, más fuertes. Ninguna de estas convicciones o visiones del deporte se verá truncada al entrar en los debates propuestos por la ética del discurso, lo que se les pedirá es que acepten ciertas reglas para que éste sea posible y conduzca a un verdadero acuerdo. Sólo así, el futuro del deporte se irá decidiendo poco a poco, y pasando de un estado de cosas peor a otro mejor-al menos si se siguen las reglas del juego discursivo.

Como afirmé en el apartado anterior, no todos los debates dentro de la comunidad deportiva se desarrollan exclusivamente de un modo dialógico, sino también práctico, es decir, a través de la acción. En este sentido, un caso de debate de este tipo que debemos tratar de convertir en más plural lo encontramos en nuestra liga de fútbol profesional. Las diferencias económicas entre los diversos clubs impiden que los equipos con menos poder económico puedan "plantear sus argumentos en el campo". Utilizando esta expresión en el sentido de utilizar las tácticas que deseen, por ejemplo, salir al ataque. En cambio, tienen que amoldarse al "argumento del fuerte", aceptar que son los débiles y amoldarse al discurso futbolístico de los poderosos. Así, por ejemplo, todos los equipos pequeños han de jugar encerrados, a la defensiva, sin poder proponer su fútbol de un modo abierto y propio. Una ordenación más justa de las relaciones de poder y de las estructuras que dan forma a nuestra liga de fútbol permitiría una situación práctica más abierta y plural en la que cada uno podría proponer sus propios argumentos futbolísticos. Este sería el objetivo de un verdadero fair play económico, no como el actual que sólo perpetua el poder y dominación de los argumentos de los más poderosos. La ética del discurso lucha contra estas desigualdades. 
La propuesta de comunidad comunicativa deportiva aquí planteada es mucho más inclusiva y extensa que la de Morgan. Tal y como afirman tanto este autor como Sebastián Solanes y Robert L. Simon, la práctica deportiva y su comunidad deben concebirse de un modo más dialógico y comunicativo. Sin embargo, con esta afirmación no basta, es necesario aclarar dos cosas: primero, cómo tornar en más comunicativa esta práctica y, segundo, quienes deben realizar esta tarea y a través de qué tipo de diálogo. Como hemos visto, el principio de la ética discursiva es el criterio a seguir para lograr una comunidad deportiva mejor, en el sentido de más comunicativa. Por otro lado, todos los afectados por las consecuencias de lo que acontece en el deporte deben ser tenidos en cuenta, sabiendo, además, que estos debates no siempre se llevan a cabo a través de palabras, sino que también pueden darse de modo práctico: a través del movimiento corporal, la lucha, y la competición.

\section{Conclusión: la ética discursiva, si está bien interpretada, es fructífera en filosofía del deporte}

La motivación principal de este artículo es la de mostrar que deberían fomentarse las propuestas que traten de integrar la filosofía anglo-americana, en cuyo seno nació la filosofía del deporte, y la continental. Para lograr este objetivo se ha analizado la aplicación que algunos autores dentro de la filosofía del deporte han hecho de una propuesta filosófica eminentemente continental como la ética del discurso. Así pues, se ha mostrado cómo Raúl Sebastián Solanes, Robert L. Simon, y William J. Morgan han propuesto concebir la comunidad deportiva según el ideal de la comunidad ideal de habla de la ética del discurso.

La propuesta de una comunidad deportiva más comunicativa se ha mostrado como muy fructífera para los fines de la ética y la filosofía del deporte. Sin embargo, tal y como se ha defendido en este artículo, ninguno de los autores que la ha planteado con anterioridad ha logrado esbozarla de un modo completo. Sin bien Sebastian Solanes la ha planteado en los términos adecuados, es decir, los de la ética del discurso propuesta por el filósofo alemán Karl Otto Apel, le ha faltado concretar cómo dicha comunidad comunicativa debe realizarse y quién debe hacerlo. Por su parte, Morgan sí ha tratado de ser más concreto a la hora de esbozar el modo en que puede lograrse una comunidad deportiva más comunicativa. Sin embargo, su deseo de salvaguardar el internalismo a toda costa le llevó a malinterpretar el pensamiento de la propuesta dialógica de Jürgen Habermas y, con ello, a pensar una comunidad deportiva comunicativa demasiado encerrada en sí misma y poco extensa, especialmente, teniendo en cuenta lo global del fenómeno deportivo en nuestro mundo actual.

Al interpretar de un modo crítico, sobre todo, las propuestas de Sebastián Solanes y de Morgan este artículo ha defendido la idea de una comunidad comuni- 
cativa en el ámbito deportivo mucho más inclusiva, más al estilo de la defendida por Simon, que la propuesta por aquellos dos, pues introduce dentro de ella no sólo a todos los implicados por las consecuencias de las acciones que acontecen en el deporte, sino también porque contempla la posibilidad de que el debate se desarrolle de modo práctico, es decir, a través de las decisiones y acciones de aquellos que lo practican. Éstas deben ser correctamente interpretadas para comprender cuáles son los valores, principios y elementos regulativos que se esconden en el trasfondo de nuestro deporte. De este modo, la comunidad comunicativa que aquí se presenta es mucho más inclusiva que las de los dos modelos teóricos aquí discutidos, lo cual permite captar de un modo mejor la complejidad que rodea a una práctica social tan central para comprender nuestra sociedad contemporánea como el deporte.

\section{Referencias bibliográficas}

Apel, K. O. (1986): Diskurs und Verantwortung. Suhrkamp: Fráncfort del Meno. BERnsteIn, R. J. (1982): "What is the Difference that makes a Difference? Gadamer, Habermas, and Rorty" In PSA: Proceedings of the Biennial Meeting of the Philosophy of Science Association 1982: pp. 331-359.

Bernstein, R. J. (1985): "Philosophy in the Conversation with Mankind." In Hermeneutics and Praxis, R. Hollinger (Ed.). Indiana, University of Notre Dame Press, pp. 54-88.

Corlett, A. (2013): "Coping with Doping" en Journal of the Philosophy of Sport, 40 (1), pp. 41-64.

Cortina, A. (2008): La Escuela de Fráncfort. Madrid: Síntesis.

CortinA, A. (2010): Justicia Cordial. Madrid: Tecnos.

Domingo Moratalla, A. (1999): "Esperanzas de Libertad: Ética y Política en la

Hermenéutica de Gadamer y Rorty." In: Estética y hermenéutica, C. Maillard, L. E. de Santiago Guervós (Eds.). Suplemento de Contrastes, Universidad de Málaga, pp. 193-212.

Fraleigh, W. (1984): Right Actions in Sport: Ethics for Contestants. Champaign (IL): Human Kinetics.

Giulianotti, R. (2005): Sport. A Critical Sociology. Cambridge: Polity Press.

Habermas, J. (1998) Facticidad y Validez. Sobre el derecho y el Estado democrático de derecho en términos de teoría del discurso. Madrid: Trotta.

KretchmaR, R.S. (2013): "Dualism, Dichotomies and Dead Ends: Limitations of Analytic Thinking about Sports". In Sports, Ethics, and Philosophy, 1:3, pp. 266-280.

LóPez FríAs, F.J. (2014a): La filosofía del deporte actual. Paradigmas y corrientes principales. Roma: (Qua.Pe.G.) Università degli Studi di Roma "Foto Italico". LÓPEZ FRÍAS, F.J. (2014b): "William J. Morgan's 'conventionalist internalism' 
approach. Furthering internalism? A critical hermeneutical response", en Sport, Ethics and Philosophy. [En Prensa]

LóPEZ FríAS, F.J. (2014c): "La filosofía del deporte. Análisis de un paradigm", en Informe USA, 30, octubre, Instituto Franklin de Investigación de Estudios Norteamericanos. http://www.institutofranklin.net/sites/default/files/files/ Informe $\% 20$ USA $\% 2030 \% 201014(1)$.pdf

Marmor, A. (2009): Social Conventions: From Language to Law. Princeton, N.J, USA: Princeton University Press.

McFeE, G. (2004): "Normativity, Justification, and (MacIntyrean) Practices: Some Thoughts on Methodologyfor the Philosophy of Sport", en Journal of the Philosophy of Sport, 31 (1), pp. 15-33.

McFee, G. (2009): "The Intrinsic Value of Sport. A reply to Culbertson", en Sport, Ethics, and Philosophy, 3 (1), pp. 19-29.

Møller, V. (2009): The Ethics of Doping and Anti-Doping: Redeeming the Soul of Sport? Nueva York: Routledge.

Morgan, W. J. (1994): Leftist Theories of Sport: A critique and reconstruction. Champaign: University of Illinois Press.

Morgan, W. J. (2002): "Social Criticism as Moral Criticism: A Habermasian Take on Sports", en Journal of Sport \& Social Issues, 26 (3), pp. 281-299.

Morgan, W. J. (2006): Why Sports Morally Matter, New York, Routledge.

Morgan, W.J. (2012): "Broad Internalism, Deep Conventions, Moral Entrepeneurs, and Sport". In Journal of the Philosophy of Sport, 39 (1), pp. 65-100.

Sebastian Solanes, R. (2012): "La ética del deporte en Karl-Otto Apel: Ética del discurso y compromiso trascendental claves para un deporte ético". In Diálogo Filosófico, 83, pp. 73-92.

SimON, R. L. (1991): Fair Play: sports, values, and society. Boulder: Westview Press.

SimOn, R.L. (2014). "From Ethnocentrism to Realism: Can Discourse Ethics Bridge the Gap?", en Journal of the Philosophy of Sport, 31 (2), pp. 122-141.

SCHNEIDER, A. J. \& Butcher, R. B. (200): "A philosophical overview of the arguments on banning doping in sport”, pp. 187 y 195, en Torbjörn Tännsjö y Claudio Tamburrini (Eds.) Values in sport: Elitism, nationalism, gender equity and the scientific manufacture of winners, New York, E\&F Spon, pp. 185199.

WitTGenstein, L. (2000): Sobre la certeza. Barcelona: Gedisa.

Francisco Javier López Frías

Universidad de Valencia

Francisco.Javier.Lopez@uv.es 